\title{
Retrospective Review of Propofol versus Etomidate during Rapid Sequence Intubation in the Emergency Department at a Tertiary Academic Center
}

\author{
Megan A. Rocchio ${ }^{1 *}$, Katelyn Sylvester ${ }^{1}$, Nahal Beik ${ }^{2}$, Nicole M. Glasser ${ }^{3}$, Paul M. Szumita1 \\ ${ }^{1}$ Department of Pharmacy, Brigham and Women's Hospital, MA, USA \\ ${ }^{2}$ Center for Drug Policy, Partners Health Care, MA, USA \\ ${ }^{3}$ Department of Pharmacy, Crozer-Keystone Health System, PA, USA \\ Email: *mrocchio@partners.org, kwsylvester@partners.org, nbeik@partners.org, nicole.glasser@crozer.org, \\ pszumita@partners.org
}

How to cite this paper: Rocchio, M.A., Sylvester, K., Beik, N., Glasser, N.M. and Szumita, P.M. (2017) Retrospective Review of Propofol versus Etomidate during Rapid Sequence Intubation in the Emergency Department at a Tertiary Academic Center. Pharmacology \& Pharmacy, 8, 231-241. https://doi.org/10.4236/pp.2017.87016

Received: November 3, 2016

Accepted: July 14, 2017

Published: July 17, 2017

Copyright $\odot 2017$ by authors and Scientific Research Publishing Inc. This work is licensed under the Creative Commons Attribution International License (CC BY 4.0).

http://creativecommons.org/licenses/by/4.0/

\begin{abstract}
Background: Etomidate is the standard induction agent used during rapid sequence intubation (RSI) in the emergency department (ED). Etomidate shortages require providers to utilize alternative agents. The purpose of this study is to compare the safety and procedural outcomes of propofol and etomidate for RSI in the ED. Methods: This was a retrospective chart review of adult patients in the ED who received propofol or etomidate for induction during RSI. The main endpoint was hypotension, defined as a systolic blood pressure $<90 \mathrm{mmHg}$ or diastolic blood pressure $<60 \mathrm{mmHg}$, within the first hour of intubation. Time to intubation, intensive care unit length of stay, hospital length of stay, and in-hospital mortality were also evaluated. Results: Two hundred and seventy five patient charts were reviewed. Of the 98 patients included, 43 patients received propofol and 55 patients received etomidate. Propofol was associated with an increased incidence of hypotension within the first hour of intubation ( $65.1 \%$ vs. $25.5 \%, \mathrm{p}<0.001)$. No difference was found in the time to intubation: $\leq 5$ minutes $(51.2 \%$ vs. $34.6 \%, p=0.83)$. The mortality rate was $11.6 \%$ in the propofol group and $27.3 \%$ in the etomidate group $(p=0.004)$. There was no difference in hospital or intensive care unit length of stay between the propofol and etomidate groups (7.7 vs. 9.2 days, $p$ $=0.23 ; 4.2$ vs. 6.3 days, $\mathrm{p}=0.31)$. Conclusion: Propofol was a safe and procedurally effective induction agent for RSI. Compared to etomidate, it was associated with an increased rate of hypotension within the first hour of intubation, with no difference in the percent of patients that required an intervention.
\end{abstract}




\section{Keywords}

Rapid Sequence Intubation, Etomidate, Zropofol, Safety, Efficacy

\section{Introduction}

Rapid sequence intubation (RSI) is the method in which pharmacological agents along with procedural methods are used in quick succession to secure the airway of critically ill patients requiring emergent endotracheal intubation [1]. The purpose of facilitating RSI with pretreatment, sedation, and neuromuscular blocking agents is to effectively secure a patient's airway while minimizing negative outcomes such as aspiration, pain, cervical spine movement, and increased intracranial and intraocular pressure associated with the initiation of intubation [1] [2]. It is important to emphasize that induction with sedation agents must occur before paralyzing patients to avoid consciousness during the procedure.

The choice of an induction agent should be based on the adverse effect profile of the drug and clinical presentation of the patient. Etomidate, a gamma-aminobutyric acid (GABA) agonist is commonly used as an induction agent administered to facilitate a state of unconsciousness [3] [4]. It has an onset of action within 10 - 20 seconds and duration of action of 4 to 10 minutes [5]. While etomidate has limited hemodynamic effects making it a desirable choice for quick sedative effects, it does possess unwanted side effects that can be dangerous for certain patient populations [6]. Its inhibition of 11- $\beta$-hydroxylase to convert cholesterol to cortisol can lead to adrenal insufficiency and caution should be taken in cases of septic shock with adrenal insufficiency due to controversial data regarding higher mortality rates associated with etomidate administration [7]-[14].

The onset of action of a bolus dose of propofol is within 10 - 50 seconds, with effects lasting between 3 - 10 minutes [15]. Although propofol is associated with severe bradycardia and hypotension, it is an optimal choice in hemodynamically stable patients with traumatic brain injuries and reactive airway disease because it does not affect cerebral perfusion and can assist in bronchodilation [15]. Other agents described in the literature, but less commonly seen in clinical practice for this indication include ketamine and midazolam [1] [2].

In many institutions, etomidate has been the standard induction agent used during RSI procedures in the emergency department (ED). Recent drug shortages have limited etomidate availability and required healthcare professionals to consider alternative agents, such as propofol, for RSI. At our institution, a steady supply of etomidate became unavailable starting in February of 2012 and did not return to adequate levels until June of 2012. Although propofol has been described in the literature for procedural sedation in the ED there are limited data regarding its use for RSI [15] [16] [17] [18]. Despite the common use of these agents during RSI, the safety and efficacy of these two agents has not been compared in randomized controlled trials. 
This retrospective review evaluates the safety and procedural outcomes of propofol compared to etomidate for induction during RSI in the ED at a large tertiary academic medical center. The main objective of our study was to determine the rate of hypotension within one hour of administration of the induction agent.

\section{Materials and Methods}

We performed a single-center, retrospective chart review of adult patients who received propofol between February 1, 2012 and May 31, 2012 (Phase I, shortage period where adequate supply of etomidate was unavailable) and those who received etomidate between September 1, 2012 and December 31, 2012 (Phase II) for induction during RSI in the ED. The protocol was reviewed and approved by the Institutional Review Board prior to data collection.

This study was performed at a tertiary academic urban level one trauma center with a 60-bed ED. The ED was provided with kits that contained all pharmacological agents needed to facilitate RSI. Etomidate was the standard drug stocked in the RSI kits as an induction agent. Due to the national medication shortage announced by the FDA in February 2012, etomidate vials were replaced with propofol. Limited etomidate vials were still available in the automatic dispensing cabinets located in the ED.

All adult patients in the ED requiring intubation who received an induction agent during RSI were eligible for enrollment. Patients were identified and included through pharmacy charging records based on RSI kit charges. Patients were excluded if they were $<18$ years old, alternative agents were used for induction (i.e. ketamine, midazolam), the RSI kits were charged twice, intubation was not required, the patient was intubated prior to arrival, or charting information was incomplete or unavailable. Patients who received any alternative agent for induction agent either than propofol during Phase I or etomidate during Phase II were also excluded.

The choice of induction agent was selected by the attending physician and emergency medicine team based on the clinical presentation of the patient. The drug shortage affecting the supply of etomidate began prior to Phase I of this investigation. During Phase I, propofol was the agent of choice for RSI by ED physicians because of the limited availability of etomidate. Propofol efficacy and safety data was reviewed during a 4-month time period of the etomidate shortage. Although limited inventory of etomidate became available in August 2012, etomidate was not considered readily available until September 1, 2012. During Phase II, both etomidate and propofol were available options, however only etomidate safety and procedural outcomes data was reviewed from September 1, 2012 through December 31, 2012 as product availability returned to adequate levels.

Data collection was processed collected through an extensive chart review of all patients who received a single dose of either etomidate or propofol prior to intubation during the respective time periods. Demographic information col- 
lected included; age, sex, height, weight, Acute Physiology and Chronic Health Evaluation (APACHE) II, and past medical history. All RSI medications, vasopressors, intravenous fluids, and antibiotics administered were also recorded. Procedure duration, complications (i.e. oral trauma), and indication for intubation were obtained from hospital procedural documents in the chart. Procedure duration was defined as $\leq 5$ minutes or $>5$ minutes to completion of the intubation. Indications for RSI were 1) airway protection, 2) respiratory failure, 3) respiratory arrest, and 4) other.

Vital signs monitored in the ED included blood pressure, heart rate, mean arterial pressure, respiratory rate, temperature, pulse oximetry and oxygenation requirements. Measurements were collected at ED admission and recorded through one hour immediately following the completed intubation. After completion of the procedure, time to extubation was defined as 1) $\leq 24$ hours, 2) $>24$ hours, and 3) $\geq 48$ hours. Other data collected included hospital length of stay, intensive care unit (ICU) length of stay, and mortality.

The main outcome assessed was hypotension within one hour of induction agent administration. Hypotension was defined as a systolic blood pressure $<90$ $\mathrm{mmHg}$ or a diastolic blood pressure $<60 \mathrm{mmHg}$. Minor outcomes assessed included time to intubation, time to extubation, ICU length of stay, hospital length of stay, and in-hospital mortality.

Continuous variables were assessed using Students t-test or Wilcoxon rank sum and non-continuous variables were assessed using Fisher's exact or Chi Squared Test when appropriate. All $\mathrm{p}$ values were two tailed and statistically significant at $\leq 0.05$.

\section{Results}

Two hundred and seventy five patient charts were reviewed. After exclusion, 98 patients met the inclusion criteria in which 43 patients received propofol and 55 patients received etomidate. A description of the enrollment and exclusions can be found in Figure 1. Overall, the groups were similar in age, sex, baseline comorbidities, and APACHE II scores. Patient demographics are outlined in Table 1.

The most common indication for intubation was airway protection. Nineteen patients in the propofol group and 14 patients in the etomidate group needed airway protection, respectively $(44.1 \%$ vs. $67.3 \%, p=0.02)$. There was no difference between the selection of neuromuscular blocking agents administered between the groups, but succinylcholine was the most popular agent administered in both groups $(60.5 \%$ vs. $61.8 \%, \mathrm{p}<0.99)$ (Table 2$)$.

The main outcome of hypotension within one hour of administration of the induction agent occurred in 28 patients in the propofol group and 14 patients in the etomidate group ( $65.1 \%$ vs. $25.5 \%, \mathrm{p}<0.0001)$. Of the 42 patients that had recorded hypotension, only 27 patients needed an intervention to reverse the episode (67.8\% in the propofol group vs. $57.1 \%$ in the etomidate group, $\mathrm{p}=0.51$ ). Interventions to reverse the hypotensive episode included additional intravenous fluid boluses, initiation of vasopressor agents, or a combination of the two. 


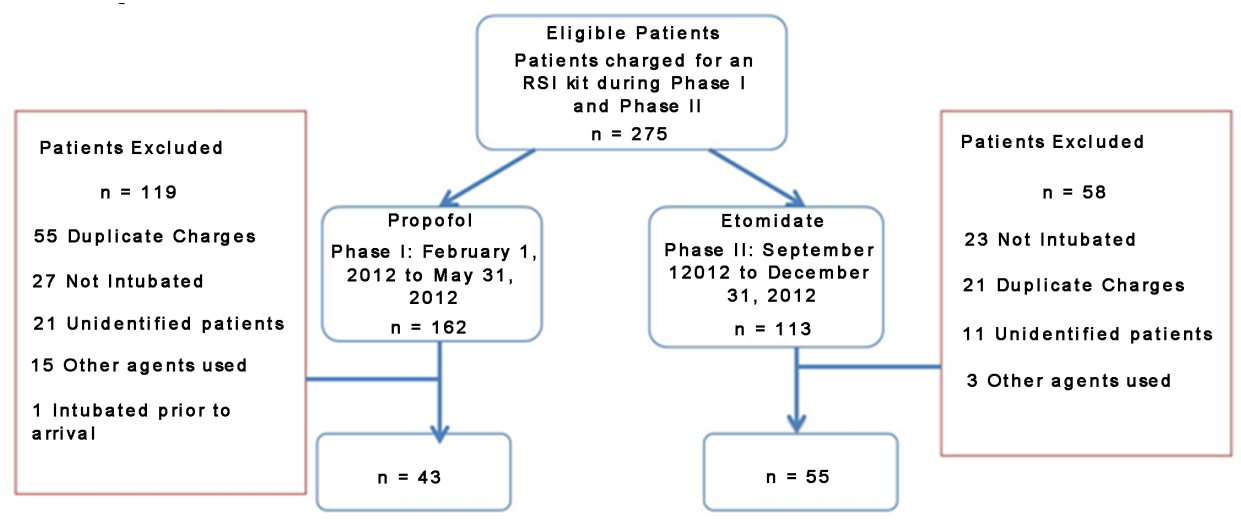

Figure 1. Patient enrollment.

Table 1. Characteristics of the study subjects.

\begin{tabular}{|c|c|c|c|}
\hline Patient characteristics & Propofol $(n=43)$ & Etomidate $(\mathrm{n}=55)$ & p-value \\
\hline Age, yrs ${ }^{1}$ & $57 \pm 18.2$ & $63 \pm 17.2$ & 0.12 \\
\hline Male sex, n (\%) & $19(44.2)$ & $24(43.6)$ & 0.31 \\
\hline Height $(\mathrm{cm})^{1}$ & $169.2 \pm 26.9$ & $144.3 \pm 61.3$ & 0.15 \\
\hline Weight $(\mathrm{kg})^{1}$ & $74.8 \pm 18.6$ & $74.1 \pm 29.5$ & 0.57 \\
\hline APACHE II $^{2}$ & $22(15-26)$ & $21(17-25)$ & 0.23 \\
\hline \multicolumn{4}{|l|}{ Comorbidities } \\
\hline Hypertension & $27(62.7)$ & $42(76.3)$ & 0.18 \\
\hline Diabetes & $11(25.5)$ & $19(34.5)$ & 0.38 \\
\hline Malignancy & $13(30.2)$ & $17(30.9)$ & 0.99 \\
\hline COPD & $2(4.6)$ & $6(10.9)$ & 0.45 \\
\hline $\mathrm{CHF}$ & $5(11.6)$ & $9(16.3)$ & 0.57 \\
\hline Tobacco smoker & $11(25.5)$ & $6(10.9)$ & 0.06 \\
\hline Abnormal LFTs & $7(16.3)$ & $11(20.0)$ & 0.79 \\
\hline Cirrhosis & $4(9.3)$ & $7(62.7)$ & 0.75 \\
\hline ESRD & $3(6.9)$ & $6(12.7)$ & 0.72 \\
\hline Prior intubations & $1(2.3)$ & $0(0)$ & 0.43 \\
\hline \multicolumn{4}{|l|}{ Baseline laboratory tests } \\
\hline SCr & $1.1 \pm 0.5$ & $2.3 \pm 0.6$ & 0.25 \\
\hline BUN & $25.1 \pm 17.3$ & $26.7 \pm 18.9$ & 0.64 \\
\hline WBC & $12.2 \pm 5.6$ & $11.9 \pm 5.1$ & 0.84 \\
\hline $\mathrm{pH}$ & $7.3 \pm 0.1$ & $7.3 \pm 0.2$ & 0.27 \\
\hline \multicolumn{4}{|l|}{ Baseline vital signs } \\
\hline Heart rate & $102.9 \pm 32.9$ & $93.9 \pm 25.5$ & 0.08 \\
\hline Respiratory rate & $20.7 \pm 7.5$ & $21.1 \pm 6.8$ & 0.10 \\
\hline $\mathrm{SBP}(\mathrm{mmHg})$ & $139.2 \pm 29.7$ & $136.8 \pm 33.6$ & 0.71 \\
\hline $\mathrm{DBP}(\mathrm{mmHg})$ & $74.4 \pm 20.5$ & $71.7 \pm 18.4$ & 0.50 \\
\hline MAP & $95.9 \pm 22.2$ & $92.9 \pm 21.0$ & 0.51 \\
\hline Temperature (F) & $97.9 \pm 2.0$ & $97.7 \pm 1.7$ & 0.10 \\
\hline
\end{tabular}

${ }^{1}$ Mean \pm Standard Deviation; ${ }^{2}$ Median (Interquartile Range); $\mathrm{SCr}=$ serum creatinine; $\mathrm{BUN}=$ blood urea nitrogen; $\mathrm{WBC}=$ white blood count; $\mathrm{SBP}=$ systolic blood pressure; $\mathrm{DBP}=$ diastolic blood pressure; $\mathrm{MAP}=$ Mean Arterial Pressure. 
Table 2. Main results of rapid sequence intubation procedure.

\begin{tabular}{cccc}
\hline Results & Propofol $(\mathbf{n}=43)$ & Etomidate $(\mathbf{n}=\mathbf{5 5})$ & p-value \\
\hline Indication, $\mathbf{n}(\%)$ & & & \\
\hline Airway protection & $19(44.1)$ & $37(67.3)$ & 0.02 \\
Respiratory failure & $18(41.9)$ & $14(25.5)$ & 0.12 \\
Respiratory arrest & $6(14)$ & $2(3.6)$ & 0.13 \\
Other $^{1}$ & $0(0)$ & $2(3.6)$ & 0.50 \\
\hline Succinylcholine & Neuromuscular blocking agents, $\mathbf{n}(\%)$ & 0.99 \\
Rocuronium & $26(60.5)$ & $34(61.8)$ & 0.99 \\
Vecuronium & $16(37.2)$ & $21(38.1)$ & 0.43 \\
Induction agents & $1(2.3)$ & $0(0)$ & \\
Dose (mg/kg) & $1.1 \pm 0.7$ & & \\
\hline
\end{tabular}

${ }^{1}$ PEA arrest; septic shock; ${ }^{2}$ Mean \pm Standard Deviation.

There was no difference in the rate of fluid or vasopressor administration between the groups (Table 3 ).

The rate of failed intubations ( $4.6 \%$ vs. $5.5 \%$ ) and oral trauma ( $2.3 \%$ vs. $3.6 \%)$ was not found to be statistically significant between the propofol and etomidate groups respectively. The mean hospital length of stay was 7.7 and 9.2 days and ICU length of stay was 4.7 and 6.3 days in the propofol compared to etomidate group. The recorded rate of mortality was $11.6 \%$ in the propofol group compared to $27.3 \%$ in the etomidate group $(p=0.004)$ (Table 4$)$.

\section{Discussion}

In this retrospective chart review analysis comparing propofol and etomidate for induction during RSI, we found that both agents have similar safety and procedural outcomes profiles. When reviewing the charts for RSI procedural records, all medications, such as fentanyl, lidocaine and/or midazolam for pretreatment were appropriately dosed and administered before the RSI procedure. Both agents had similar rates of oral trauma, failed intubations, time to intubation, and time to extubation. The mean dose of propofol was $1.1 \mathrm{mg} / \mathrm{kg}$ and etomidate was $0.26 \mathrm{mg} / \mathrm{kg}$, which are slightly lower than the recommended doses for induction during RSI [5] [15]. There were no records of additional adverse events recorded in the chart review related to the administration of either induction agent.

As expected, propofol had a higher rate of hypotension within the first hour after the induction agent was administered. Although propofol had a significantly higher rate of hypotension compared to etomidate $(65.1 \%$ vs. $25.5 \%, \mathrm{p}<$ $0.0001)$, there was no difference in the number of patients who required an intervention to address these events and all episodes were recorded without longterm adverse events. Of those patients in each group that did require an 
Table 3. Outcome results.

\begin{tabular}{cccc}
\hline Results & Propofol $(\mathrm{n}=\mathbf{4 3})$ & Etomidate $(\mathrm{n}=55)$ & p-value \\
\hline Hypotension, $\leq 1$ hour, n (\%) & $28(65.1)$ & $14(25.5)$ & 0.0001 \\
\hline Results & Propofol $(\mathrm{n}=\mathbf{2 8 )}$ & Etomidate $(\mathrm{n}=14)$ & p-value \\
\hline Intervention, n (\%) & $19(67.8)$ & $8(57.1)$ & 0.51 \\
Fluids & $10(52.6)$ & $3(37.5)$ & 0.25 \\
Vasopressors & $6(33.3)$ & $3(37.5)$ & 0.99 \\
Both & $3(15.7)$ & $2(25.0)$ & 0.99 \\
\hline
\end{tabular}

Table 4. Secondary outcome results.

\begin{tabular}{|c|c|c|c|}
\hline Results & Propofol $(n=43)$ & Etomidate $(n=55)$ & $\mathrm{P}$-value \\
\hline Oral trauma, n (\%) & $1(2.3)$ & $2(3.6)$ & 0.99 \\
\hline Failed extubation, $\mathrm{n}(\%)$ & $2(4.6)$ & $3(5.5)$ & 0.99 \\
\hline \multicolumn{4}{|c|}{ Time to intubation, $\mathrm{n}(\%)$} \\
\hline$\leq 5$ minutes & $22(51.2)$ & $30(34.6)$ & 0.83 \\
\hline$>5$ minutes & $21(48.8)$ & $25(45.4)$ & 0.84 \\
\hline \multicolumn{4}{|c|}{ Time to extubation, $\mathrm{n}(\%)$} \\
\hline$\leq 24$ hours & $13(30.2)$ & $10(19.6)$ & 0.23 \\
\hline$>24$ hours $\leq 48$ hours & $16(37.2)$ & $17(33.3)$ & 0.52 \\
\hline$\geq 48$ hours & $14(32.6)$ & $24(47.1)$ & 0.30 \\
\hline Hospital LOS ${ }^{1}$ & $7.7 \pm 6.3$ & $9.2 \pm 7.8$ & 0.31 \\
\hline ICU LOS ${ }^{1}$ & $4.7 \pm 5.1$ & $6.3 \pm 7.1$ & 0.22 \\
\hline Mortality, n (\%) & $5(11.6)$ & $20(27.3)$ & 0.004 \\
\hline
\end{tabular}

${ }^{1}$ Mean \pm Standard Deviation

intervention, they received fluid boluses with sodium chloride $0.9 \%$, vasopressor agents (norepinephrine, phenylephrine, vasopressin), or both. For patients that were already receiving fluids or vasopressor agents before initiation of RSI and had a recorded hypotensive event within one hour after administration of the induction agents, increases in the rate of the vasopressor agents or additional fluid boluses were considered an intervention.

Some hypotensive events might have been associated with fluctuations in ventilator settings, the use of continuous sedative infusions for sedation (i.e. propofol, midazolam) or other undocumented physiological changes unrelated to the induction agent administered. This could explain the resolution of hypotension in the 15 (35.6\%) patients with a documented hypotensive event that did not require additional intravenous fluids or initiation of a vasopressor. Unfortunately, this hypothesis cannot be confirmed due to the fact that not all charts had recorded ventilator settings or documented titration of continuous sedation 
agents. The majority of the charts only confirmed the initial ventilator settings and rate of continuous sedation immediately after the placement of the endotracheal tube.

The overall mortality rate was $25.5 \%$ in both the propofol and etomidate groups combined. The mortality rate recorded in the etomidate group was higher compared to the propofol group $(27.3 \%$ vs. $11.6 \%, \mathrm{p}<0.004)$. We found that a large majority of patients in both groups needed to be intubated for airway protection $(44.1 \%$ vs. $67.3 \%, \mathrm{p}<0.025)$. After looking at the population demographics in more detail of each patient intubated for airway protection, more patients in the etomidate group were admitted to the ED for a traumatic brain injury and intracerebral hemorrhages. Although the severity of illness is comparable in the two groups, it can be presumed that the patient population in the etomidate group had a higher baseline mortality risk based on the requirement for hospital admission and clinical presentation. It has been reported in previous literature that etomidate can be associated with increased risk of death after a single dose, but this cannot be correlated to our investigation.

\section{Limitations}

There are several limitations in this study. This was a single-center, retrospective, observational chart review with a small patient sample size to analyze based on the timing of the national backorder of etomidate requiring a temporary switch to propofol as our preferred induction agent for RSI. Incomplete patient charts resulted in unavailable or missing pertinent information. The main outcome of hypotension within the first hour of induction might have been associated with fluctuations in ventilator settings, continuous infusions of sedatives, or other undocumented physiological changes unrelated to the induction agent administered. Unfortunately, only the initial ventilator settings and dose of sedation medications were documented in the ED charts, making it unfeasible to correlate any hypotensive episodes or hemodynamic instability to ventilation or additional concomitant medications.

Regarding our statistical analysis, the secondary outcome of in-hospital mortality did not include a multivariate test for associated risk factors for increased mortality risks. Since this was not analyzed, higher rates of mortality in the etomidate group compared to propofol cannot be directly correlated to its administration.

\section{Conclusion}

Propofol was associated with an increased rate of hypotension within the first hour of intubation; however, there was no difference in the percent of patients that required an intervention to treat the hypotensive event. Other endpoints suggest there were no further clinically significant differences between the two groups in adverse events or failed intubations. It appears that both of these pharmacological agents are similar in safety and procedural outcomes for use in RSI in the ED. The increased mortality rate found with etomidate warrants fur- 
ther analysis comparing these two agents during RSI.

\section{Ethics Approval and Consent to Participate}

The protocol was reviewed and approved by the Brigham and Women's Hospital/Partners Institutional Review Board prior to data collection (Protocol \#: 2012p-002126). Consent to participate was waived as data was collective retrospectively.

\section{Consent for Publication}

Consent for publication is not applicable as this study as there is no individual patient data or information.

\section{Availability of Data and Materials}

All data generated during and/or analyzed during the current study are included in this published article.

\section{Competing Interests}

M.A.R., K.S., N.B., N.M.G., P.M.S. declare no competing interests.

\section{Funding}

No financial support or funding was obtained for this analysis. M.A.R., K.S., N.B., N.M.G., P.M.S. do not report any affiliation with or financial interest in a commercial organization that poses a conflict of interest with this article.

\section{Authors' Contributions}

M.A.R., K.S., N.B. developed the project's design. N.M.G. retrospectively collected all data points. M.A.R., K.S., N.B., N.M.G., P.M.S. interpreted the results and contributed to the writing of the manuscript.

\section{References}

[1] Mace, E. (2008) Challenges and Advances in Intubation: Rapid Sequence Intubation. Emergency Medicine Clinics of North Americ, 26, 1043-1068. https://doi.org/10.1016/j.emc.2008.10.002

[2] Hampton, J.P. (2011) Rapid-Sequence Intubation and the Role of the Emergency Department Pharmacist. American Journal of Health-System Pharmacy, 68, 13201330. https://doi.org/10.2146/ajhp100437

[3] Skinner, H.J., Biswas, A. and Mahajan, R.P. (1998) Evaluation of Intubating Conditions with Rocuronium and Either Propofol Or Etomidate for Rapid Sequence Induction. Anaesthesia, 53, 702-706. https://doi.org/10.1046/j.1365-2044.1998.396-az0506.x

[4] Mallon, W.K., Keim, S.M., Shoenberger, J.M. and Walls, R.M. (2009) Rocuronium vs. Succinylcholine in the Emergency Department: A Critical Appraisal. Journal of Emergency Medicine, 37, 183-188. https://doi.org/10.1016/j.jemermed.2008.07.021

[5] No Authors (2004) Etomidate Package Insert. Hospira, Inc., Lake Forest, IL.

[6] Nestor, N.B. and Burton, J.H. (2008) ED Use of Etomidate for Rapid Sequence In- 
duction. American Journal of Emergency Medicine, 26, 946-950.

https://doi.org/10.1016/j.ajem.2007.12.002

[7] Edwin, S.B. and Walker, P.L. (2010) Controversies Surrounding the Use of Etomidate for Rapid Sequence Intubation in Patients with Suspected Sepsis. Annals of Pharmacotherapy, 44, 1307-1313. https://doi.org/10.1345/aph.1M664

[8] Jung, B., Clavieras, N., Nougaret, S., et al. (2012) Effects of Etomidate on Complications Related to Intubation and on Mortality in Septic Shock Patients Treated with Hydrocortisone: A Propensity Score Analysis. Critical Care, 16, R224. https://doi.org/10.1186/cc11871

[9] Lundy, J.B., Slane, M.L. and Frizzi, J.D. (2007) Acute Adrenal Insufficiency after a Single Dose of Etomidate. Journal of Intensive Care Medicine, 22, 111-117. https://doi.org/10.1177/0885066606298140

[10] Hohl, C.M., Kelly-Smith, C.H., Yeung, T.C., et al. (2010) The Effect of a Bolus Dose of Etomidate on Cortisol Levels, Mortality, and Health Services Utilization: A Systematic Review. Annals of Emergency Medicine, 56, 105-113. https://doi.org/10.1016/j.annemergmed.2010.01.030

[11] Hildreth, A.N., Mejia, V.A., Maxwell, R.A., et al. (2008) Adrenal Suppression Following a Single Dose of Etomidate for Rapid Sequence Induction: A Prospective Randomized Study. The Journal of Trauma, 65, 573-579. https://doi.org/10.1097/TA.0b013e31818255e8

[12] Vinclair, M., Broux, C., Faure, P., et al. (2008) Duration of Adrenal Inhibition Following a Single Dose of Etomidate in Critically Ill Patients. Intensive Care Medicine, 34, 714-719. https://doi.org/10.1007/s00134-007-0970-y

[13] Mohammad, Z., Afessa, B. and Finkielman, J.H. (2006) The Incidence of Relative Adrenal Insufficiency in Patients with Septic Shock after the Administration of Etomidate. Critical Care, 10, R105.

[14] Mcphee, L.C., Badawi, O., Fraser, G.L., et al. (2013) Single-Dose Etomidate Is Not Associated with Increased Mortality in ICU Patients with Sepsis: Analysis of a Large Electronic ICU Database. Critical Care Medicine, 41, 774-783. https://doi.org/10.1097/CCM.0b013e318274190d

[15] No Authors (2009) Propofol Package Insert. APP Pharmaceuticals, LLC, Schaumburg.

[16] Barr, J., Egan, T., Sandoval, N., et al. (2001) Propofol Dosing Regimens for ICU Sedation Based upon an Integrated Pharmacokinetic-Pharmacodynamic Model. Anesthesiology, 95, 324-333.

[17] Black, E., Campbell, S.G., Magee, K. and Zed, P.J. (2013) Propofol for Procedural Sedation in the Emergency Department: A Qualitative Systematic Review. Annals of Pharmacotherapy, 47, 856-868. https://doi.org/10.1345/aph.1R743

[18] Patanwala, A.E., Christich, A.C., Jasiak, K.D., et al. (2013) Age-Related Difference in Propofol Dosing for Procedural Sedation in the Emergency Department. The Journal of Emergency Medicine, 7, 1-6. 


\section{Abbreviations}

Rapid Sequence Intubation (RSI);

Emergency Department (ED);

Gamma-Aminobutyric Acid (GABA);

Acute Physiology and Chronic Health Evaluation (APA-CHE).

Presented at the $10^{\text {th }}$ Annual New England Critical Care Symposium, May $1^{\text {st }}$ 2013, Boston, Massachusetts; 32 $2^{\text {st }}$ Annual Eastern States Conference on May $13^{\text {th }}$-May $17^{\text {th }} 2013$ Hersey, Pennsylvania; $43^{\text {rd }}$ Annual Society of Critical Care Medicine (SCCM) Critical Care Congress on January $9^{\text {th }}$-January $13^{\text {th }} 2014$ San Francisco, California.

\section{Scientific Research Publishing}

Submit or recommend next manuscript to SCIRP and we will provide best service for you:

Accepting pre-submission inquiries through Email, Facebook, LinkedIn, Twitter, etc. A wide selection of journals (inclusive of 9 subjects, more than 200 journals)

Providing 24-hour high-quality service

User-friendly online submission system

Fair and swift peer-review system

Efficient typesetting and proofreading procedure

Display of the result of downloads and visits, as well as the number of cited articles Maximum dissemination of your research work

Submit your manuscript at: http://papersubmission.scirp.org/

Or contact pp@scirp.org 